\title{
GD Communication
}

\section{Dermocosmetics for Use in Rosacea: Guideline of the Society for Dermopharmacy}

\author{
Joachim Kresken ${ }^{\mathrm{a}} \quad$ Ursula Kindl $^{\mathrm{b}}$ Walter Wigger-Alberti ${ }^{\mathrm{c}}$ \\ Benjamin M. Clanner-Engelshofen ${ }^{d}$ Markus Reinholz ${ }^{d}$

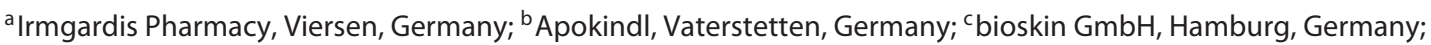 \\ ${ }^{\mathrm{d}}$ Department of Dermatology and Allergology, Ludwig-Maximilians University, Munich, Germany
}

\section{Keywords}

Guideline - Rosacea - Dermocosmetics - Efficacy - Tolerance .

Product documentation - Society for Dermopharmacy

\begin{abstract}
Rosacea is a widespread inflammatory skin disease that is chronically recurrent and affects predominately the central parts of the face. Affected individuals typically react to numerous cosmetics with redness, burning, and/or worsening of the complexion. Consequently, there is a high demand for dermocosmetics that do not provoke such reactions and are suitable for use in rosacea. The present guideline of the Society for Dermopharmacy describes the requirements that dermocosmetics for use in rosacea should meet. They include, inter alia, methods to prove the efficacy of and tolerance to these cosmetics, as well as the product documentation that the manufacturer or the distributing company should make available to professionals, like dermatologists and pharmacists, counseling patients with rosacea.
\end{abstract}

(c) 2018 S. Karger AG, Basel

\section{Introduction}

The expert panel for dermocosmetics of the Society for Dermopharmacy (GD Gesellschaft für Dermopharmazie e.V.) as an independent organization made it its task to define the minimum requirements for the quality and documentation of dermocosmetics within the framework of guidelines. The present guideline considers products provided for the skin disorder rosacea. It refers to the $S 1$ guideline "Rosacea" of the German Dermatological Society from 2013 [1], as well as to the statement "Interdisciplinary Management of Rosacea" of the Society for Dermopharmacy from 2014 [2].

Rosacea, called "copper rose" or "copper fins" in the German vernacular, is a chronic inflammatory skin disease that presents predominantly on the face and affects presumably more than 4 million people in Germany alone [1-5]. Affected patients typically react to numerous cosmetics with redness, burning, and/or worsening of the complexion. Consequently, there is a high demand for dermocosmetics that do not provoke such reactions and are suitable for use in rosacea.

Products that are considered for this particular purpose are subject to special requirements. They should be examined via proper scientific methods to ensure their

\section{KARGER}

(c) 2018 S. Karger AG, Basel

E-Mail karger@karger.com

www.karger.com/spp
Joachim Kresken

GD Gesellschaft für Dermopharmazie e.V

Gustav-Heinemann-Ufer 92

DE-50968 Cologne (Germany)

E-Mail joachim.kresken@gd-online.de 
suitability. This applies to skin cleansing, skin care, and sun protection formulations, as well as to other cosmetics. The galenic properties of these products, as well as the outcomes of the conducted studies regarding their efficacy and tolerance, should be well documented and put at the disposal of professionals, like dermatologists and pharmacists, counseling patients with rosacea.

Up to now, there has been no coherent or interdisciplinarily coordinated concept to implement those demands. This guideline aims to close this gap. It should provide assistance for professionals concerned with the development, testing, marketing, and evaluation of these products together with counseling on their correct use by patients. Furthermore, it provides guidance and advice for the selection and use of suitable products for interested consumers.

The guideline was compiled by an interdisciplinary panel of experts with the help of international literature. It applies to "standard situations" and considers the most recent scientific knowledge available for each particular question. It serves as a recommendation that requires constant reviewing and is subject to change according to new medical insights and applicability in daily practice. Its consideration does not guarantee achievement of the desired objectives. It does not claim to be complete.

\section{Definition of Dermocosmetics}

The Society for Dermopharmacy defines dermocosmetics as cosmetics in which the cosmetic purpose is achieved with regard to dermatological and pharmaceutical aspects. As such cosmetics are also used by consumers with preexisting skin defects or skin diseases, they should meet certain requirements regarding their quality and documentation, possibly exceeding the legal standards.

Dermocosmetics in accordance with this guideline are products that are offered for use in rosacea in particular. Like all cosmetic products, they are subject to regulation (EC) No. 1223/2009 on cosmetic products in all EU member states. In Switzerland, the legal basis is the food law with the food and commodity regulation and the regulation on cosmetics.

\section{Target Groups and Intention of This Guideline}

The target groups of this guideline are professionals who develop, manufacture, examine, analyze, market, or evaluate dermocosmetics for use in rosacea, as well as pro- fessionals who counsel patients and consumers who are interested in guidelines and advice for the selection and proper application of dermocosmetics for use in rosacea.

This guideline is a representation and recommendation developed by an expert panel taking into account the relevant literature. It specifies quality requirements and offers decision-making support to the persons mentioned above so that they can fulfill their tasks.

\section{Characteristics of Rosacea}

\section{Clinical Symptoms}

Rosacea is an inflammatory skin disease that is chronically recurrent and affects predominately the central parts of the face. The forehead, nose, chin, and cheeks are affected most often, but also the chest, neck, and scalp can be afflicted (extrafacial rosacea).

The cardinal symptoms of rosacea are paroxysmal erythema with heat sensation (flush), persisting erythema, papules, pustules, lymphedema, and vasodilation (telangiectasia), with the erythema often omitting the periorbital region [1]. Burning and prickling sensations are additional symptoms. Further localized or diffuse edema, as well as skin dryness, tissue thickening (phyma), and/or involvement of the ocular region, can occur.

Rosacea can emerge in different forms and with varying degrees of severity. The following forms can be distinguished $[2,5]$.

\section{Preliminary Stage (Rosacea Diathesis)}

In the currently not precisely defined preliminary stage, called rosacea diathesis, transient, paroxysmal erythema that lasts several minutes, accompanied by a feeling of heat, can occur $[1,6]$. These symptoms, also called "flushing" or "blushing," can occur in other types of rosacea with varying frequency as well.

The mentioned symptoms can be triggered by exogenous as well as by endogenous stimuli. Some exogenous trigger factors are chemical irritants, but also environmental factors like heat, cold, and solar radiation can be triggers. Endogenous factors include spicy food, alcohol, hot beverages, and psychological stress.

\section{Subtype I: Erythematotelangiectatic Rosacea}

This subtype is characterized by a lasting redness of the central areas of the face (persistent centrofacial erythema). Furthermore, more or less pronounced telangiectasia emerges. Most affected patients report paresthesia like burning, stinging, itchiness or dryness, and flaking. 
Subtype II: Papulopustular Rosacea

In this subtype a persisting centrofacial erythema with single or grouped, often symmetrically arranged, inflammatory reddened papules and pustules appear, persisting over several weeks. Furthermore, swelling in the form of a lymphedema can occur. In exceptional cases, these skin alterations can affect the chest, neck, décolleté, and/or scalp.

This subtype has to be distinguished from acne especially in young patients. An essential distinctive feature is the absence of comedones.

Subtype III: Glandular Hyperplastic Rosacea

This form is characterized by big inflammatory nodules and plaques, as well as by sebaceous gland hyperplasia, manifesting in localized phymas or diffusely. Mostly men are affected by this subtype.

Phymas can also occur together with other manifestations of rosacea and are particularly burdensome for the affected persons. They can appear on the nose (rhinophyma), the chin/jaw (gnathophyma), the forehead (metophyma), the ears (otophyma), and the eyelids (blepharophyma).

\section{Special Forms}

Besides the mentioned subtypes, there are numerous special forms of rosacea $[1,2]$. Of special clinical interest is, among others, rosacea of the eye (ophthalmorosacea), which is often missed $[7,8]$. It can appear in addition to cutaneous rosacea or in isolation.

Other special forms described in the $\mathrm{S} 1$ guideline "Rosacea" of the German Dermatological Society are rosacea fulminans as a maximal variant of the disease, steroid rosacea, rosacea conglobata, Gram-negative rosacea, rosacea in childhood, morbus Morbihan, and granulomatous rosacea $[1]$.

\section{Etiology and Pathogenesis}

The cause and pathophysiology of rosacea have not been fully established. Presumably, it is a multifactorial process [9-11]. Disorders of the innate immune system, neuroinflammatory mechanisms, UV radiation, local inflammatory reactions to cutaneous microorganisms, and alterations of vascular and possibly lymphatic vessel regulation seem to play a role [12]. As possible triggers of the inflammatory reactions, dysfunction of cathelicidins signal molecules of the innate immune system - and the presence of demodex mites on the skin, among others, have been discussed [13-15].

People suffering from rosacea often complain of recurrent episodes of flush-like redness of the face. They pos- sibly suffer from hypersensitivity against physiologic stimuli. Potential causes of this are neurogenic inflammatory reactions or the influence of mast cells [16]. As a triggering factor of early rosacea (erythematous forms), dysregulation of neuroimmunologic and neurovascular communication mechanisms is assumed $[9,12]$.

\section{Frequency and Relevance of the Disease}

The prevalence of rosacea has been examined in different studies. However, the results of these studies vary vastly. Among adult subjects, a prevalence of $2.3 \%$ was recorded [4]. Extrapolated to the total population of Germany, more than 4 million persons are affected.

Despite the disease being as limiting to the quality of life as other chronic skin diseases [17, 18], in many affected patients the diagnosis is never made. Especially the preliminary stage and the erythematotelangiectatic subtype I are underrated as a fateful predisposition to blushing [2]. The phymatous forms are misinterpreted as stigmas of alcohol abuse.

To ascertain rosacea, a medical examination, usually by a dermatologist, is always necessary. In $80 \%$ of the examined patients, the disease is diagnosed at or after the age of 30 years [19]. Women most often develop the disease beginning at the age of 35 years and have the highest prevalence at the age of 61-65 years. In men, the prevalence increases significantly after the age of 50 years [20]. Rosacea can affect persons with every skin pigmentation type, but the Celtic light-skinned type (Fitzpatrick I-II) is affected more often than the Mediterranean dark-skinned type [21].

\section{Management of Rosacea}

Due to lacking evidence, a special diet cannot be recommended $[1,2]$. Nonetheless, it is advisable to avoid trigger factors, which lead to vasodilation and flushing in the face (e.g., alcohol, spicy dishes, and hot beverages). Moreover, patients should avoid individual trigger factors as far as they can be identified in the course of the disease.

If the clinical signs of rosacea are present, affected patients should be referred to a dermatologist at an early stage. It is the job of the dermatologist to confirm the diagnosis and start an appropriate therapy. In erythematotelangiectatic rosacea and papulopustular rosacea, a topical therapy is sufficient in many cases [1, 2, 22-24]. In more severe cases and in therapy-resistant mild cases, a systemic or combined topical/systemic therapy can be necessary $[1,2,25]$. 
For the topical therapy of papulopustular rosacea, several $0.75 \%$ metronidazole formulations, as well as a $15 \%$ azelaic acid gel and, since March 2015, a 1\% ivermectin cream, are approved in Germany. The latter, applied only once daily, has been proven to be more efficient and tolerable compared to a twice-daily applied $0.75 \%$ metronidazole cream [26]. Additionally, in February 2014, a 0.5\% brimonidine tartrate gel for the treatment of flushing, which was previously only barely treatable, was approved $[2,27]$.

The only approved systemic therapy in Germany is a semiretard capsule formulation with $40 \mathrm{mg}$ doxycycline [28]. Aside from that, several drugs are prescribed offlabel, including systemic isotretinoin $[1,2,25]$. In addition to the drug therapy of telangiectasia and phymas, physical therapies like lasers or dermabrasion can be administered [2]. On the contrary, topical or systemic glucocorticoids are not indicated in rosacea since they only can worsen the disease [2]. Solely in rosacea fulminans, short-term systemic glucocorticoids are applied $[1,2]$.

The use of adequate dermocosmetics plays an important role in the management of rosacea [2, 23, 29-33]. All skin cleansing, skin care, and sun protection products, as well as additional cosmetics used during therapy or between therapy intervals, have to take into account the special needs of the skin of patients with rosacea.

\section{Formulations and Ingredients of Dermocosmetics for Use in Rosacea}

\section{Skin Cleansing Products}

Since the skin of patients with rosacea shows increased irritability and vascular reactivity, the skin cleansing process has to be very gentle. Therefore, the water should be lukewarm to avoid vascular reactions [30]. Also, intense rubbing during drying of the face, peeling products, and blood-flow-promoting facemasks, as well as tonics, which contain alcohol or other blood-flow-stimulating or astringent ingredients, have to be avoided [2, 32, 34].

As washing lotions, soap-free products with a mildly acidic $\mathrm{pH}$ value that contain no detergent with irritating potential like sodium lauryl sulfate should be used exclusively [2, 30, 32-37]. However, a disadvantage of nonirritant washing lotions is the fact that they dissolve humectants out of the skin, leading to a feeling of tension especially in the face. Because of this, for rosacea, skin cleansing products that can be applied without the use of additional water and removed with facial tissue without water are preferred. Such products are called "cleansing fluids", among other terms.

\section{Skin Care Products}

Skin care products for use in rosacea have to be geared to the needs of the skin disease in terms of their composition and their galenic properties. If this fact is considered carefully, skin care can make a substantial contribution to the management of rosacea, during drug therapy or between therapy intervals, to alleviate skin reactions like burning, stinging, and vasodilation [30, 32, 33, 36].

As a rule for facial care in rosacea, moisturizing, hydrophilic formulations with no or a minor lipid percentage, like hydrogels, hydro-dispersion gels, $\mathrm{O} / \mathrm{W}$ creams, or lamellar creams, are preferred. However, this apparently reasonable concept has been confirmed by only a few studies so far $[32,38,39]$. If a cream formulation is desired and simultaneously hypersensitivity against emulsifiers exists, one should avoid $\mathrm{O} / \mathrm{W}$ creams and prefer lamellar creams [2, 34].

Strongly lipid-emphasized formulations, especially on the basis of mineral oils, should be avoided in rosacea because they can lead to heat accumulation and worsening of the complexion $[2,32]$. Merely within the scope of a systemic therapy with isotretinoin, which leads to strong skin dehydration, the short-term use of formulations with a higher lipid percentage seems to be beneficial.

Since rosacea is triggered by UV radiation, affected individuals should use sufficient sun protection. To this end, besides the use of sun protection products (see below), UV-filter-containing skin care products with a sun protection factor of 20-30 against UVB and additional UVA protection are suitable. Such products have the advantage of being suitable for sunny days, if the sun protection factor is sufficient, with no need to switch to a typical sun protection product.

Active cosmetic ingredients in skin care products for use in rosacea play a minor role compared to dermocosmetics for other purposes. Additives of substances with skin-calming, anti-inflammatory, or vessel-stabilizing properties seem reasonable, but the benefit of such ingredients has been documented in clinical studies for only a few additives, like kinetin [40], retinaldehyde [41], and licochalcone A [42], as well as for some special mixtures of active ingredients $[43,44]$.

Skin care products with blood-flow-promoting or "cell-stimulating" ingredients, often found in antiaging products, should be avoided in rosacea $[2,34]$. The same applies for products with essential oils or substances like menthol or camphor [1]. Besides that, no products designated for use in acne or acne-prone skin should be used, despite the fact that the appearance of papulopustular rosacea might be similar. 


\section{Sun Protection Products}

Since individuals with rosacea, and erythematotelangiectatic rosacea in particular, are very sensitive to UV radiation, they have to use efficient UV protection, especially with a high degree of sun exposure. They should strictly obey the ABC rule of sun protection (avoid [the sun], blanket [unprotected body parts], [apply sun protection] cream). By "cream", the use of a sun protection product appropriate for the degree of exposure is meant.

Besides the sun protection factor, which should be denoted according to the recommendation of the EU commission of 2006 [45], another important aspect that should be ensured is an adequate base of the sun protection product. As in facial skin care (see above), hydrophilic formulations like hydro-dispersion gels ("gel creams") or $\mathrm{O} / \mathrm{W}$ creams with a low lipid percentage should be preferred. Such formulations are commonly less water and sweat resistant compared to more lipidrich products. Therefore, they have to be applied more frequently to maintain their expected protective effect.

Comparative investigations on the dermal tolerance of individual UV filters in rosacea cannot be found in the journals listed in the PubMed database. That is why the statement made by some experts in the $S 1$ guideline of the German Dermatological Society [1], i.e., that the micropigments titan dioxide and zinc oxide should be preferred, is not based on solid data.

\section{Other Products}

Besides skin cleansing, skin care, and sun protection, every other cosmetic measure in rosacea should be organized so as not to provoke skin reactions. Hence, men should avoid soap-containing shaving foams and, in case of doubt, prefer dry shaving over wet shaving. Besides that, skin-calming balms should be used instead of alcohol-containing aftershave [2].

Temperature changes from cold to warm are important triggers for rosacea. That is why cold protection creams, which are used in winter sports and professional work in cold rooms, for example, should be carefully removed with soft tissues after reentering warm rooms. Otherwise, heat accumulation and worsening of the complexion can occur [2].

To relieve the heat sensation, which is especially burdensome for many patients suffering from rosacea, some manufacturers offer thermal water sprays. These mineral rich solutions provide a refreshing effect instantaneously after application and often alleviate the redness of the skin. However, due to evaporation of the sprayed-on water, transepidermal water loss is stimulated, which is why some time after the application a feeling of tension can occur.

To conceal reddened skin patches, green-tinted skin care creams or make-up is suitable. Owing to such products, the quality of life of the users can be improved significantly [46, 47]. The base of these products should be adjusted to the skin condition and has to be free from mineral oils, fragrances, and preservatives [2,34]. The users should be informed that after the application of topical drugs the application of tinted skin care products or make-up should take place at least 10 min later.

Strong-coverage camouflage products are less suited for the use in rosacea. The main reason is that these products often contain large amounts of pigments, fats, and waxes, which are mostly only removable by intensive skin cleansing with products containing probably irritant ingredients [34].

\section{Desirable Effects and Proofs of Efficacy}

Whether a product has to provide a proof of efficacy for use in rosacea depends on its marketing. While common cosmetic effects like skin cleansing or skin care do not need a special proof of efficacy, distinct health claims like vessel stabilization, redness reduction, skin barrier stabilization, or UV protection need to be proven via appropriate methods.

All advertised additional effects have something in common, i.e., that they depend on the complete formulation and not only on the presence of a particular ingredient. Positive results of in vitro studies, performed with ingredients of a product, yield information about possible effects but do not ensure the effect in a special galenic formulation after dermal application. Therefore, controlled in vivo studies are necessary.

Placebo-controlled double-blind trials are considered the gold standard for proof of efficacy. Concerning dermocosmetics, such studies are only reasonable if the awarded effect is explicitly connected to a single ingredient. If the effect is based on the whole product, however, another study design can be used, e.g., intraindividual comparison between treated and nontreated skin areas (e.g., a split face study) or comparison of treated and nontreated groups of study participants.

In every trial design, the application period and (after calculation of the statistical power) a sufficient number of study participants should be taken into consideration. For the evaluation, based on drug trials $[27,28]$, a clinically relevant score for rosacea should be used and a stan- 
dardized evaluation of the efficacy should be performed by the study participants. Furthermore, instrumental measurements of skin hydration, transepidermal water loss, and/or redness of the skin, as well as standardized photography of the face and evaluation by trained professionals, are recommended. The latter is especially important due to the varying clinical signs occurring in rosacea.

If UV protection is claimed for a skin care product, this should be proven via the same methods used for sun protection products as recommended by the industrial association Cosmetics Europe [48]. Subsequently, to verify the UVB protection, or more specifically the SPF, the in vivo method according to EN ISO 24444:2010, and for the UVA protection the in vitro method according to EN ISO 1443:2012, should be used.

A proof of efficacy has also to be carried out if a product is directly compared and declared superior to other similar products. Whereas for proof of a superior skin care effect the same in vivo methods as for the ingredient effects could be used in principle, standardized washing tests are advantageous for comparative proofs of the efficacy of skin cleansing products [49-52]. With these methods, however, only skin cleansing products used with water can be tested. For the proof of efficacy of products used without water, however, which are preferred for cleansing of rosacea skin as noted in Formulations and Ingredients of Dermocosmetics for Use in Rosacea, standardized testing procedures have not yet been published.

\section{Undesirable Effects and Proofs of Tolerance}

The risks of dermocosmetics for use in rosacea can be intolerance reactions like acute or chronic-cumulative irritant contact dermatitis, sensory irritations, and allergic contact dermatitis based on sensitization of the delayed reaction type. Studies of the Information Network of Departments of Dermatology (Informationsverbund Dermatologischer Kliniken, IVDK) have confirmed practical experiences whereupon patients with rosacea often react with intolerance to cosmetics due to irritation $[53,54]$. In contrast, allergic contact sensitization to ingredients of cosmetics does not seem to occur in rosacea patients more often than in other groups of persons.

As with all cosmetic products, the manufacturer or the distributing company of dermocosmetics for use in rosacea needs to issue a safety report. The Notes of Guidance of the Scientific Committee on Consumer Safety (SCCS) operating on behalf of the EU commission serve as a basis for it [55]. In accordance with these notes, it can be scientifically and ethically necessary, for the safety evaluation of products offered to specific groups of consumers, to take the safety of the final product into account and not only the knowledge about the toxicity of the single ingredients.

This criterion mentioned by the SCCS applies undoubtedly also to dermocosmetics for use in rosacea. Hence, an additional safety evaluation of the final product cannot be waived. An important aspect of such a safety evaluation that needs to be considered is the risk of acute or chronic-cumulative irritant reactions, which need to be elucidated by in vivo methods.

While the risk of acute intolerance reactions can be determined by the occlusive epicutaneous patch test, the repetitive epicutaneous patch test and the Duhring chamber test are suitable to evaluate the risk of chronic-cumulative irritant reactions [56-61]. Further results regarding the irritant potential of skin cleansing products used with water can be delivered by the elbow-washing test [62] and the forearm-washing test [63]. In contrast, the few in vitro methods that have been developed to determine the irritation potential of commercial products [64-66] have not gained much acceptance so far.

The study design and the number of participants in the mentioned in vivo methods have to be chosen in a way that the statistical analysis can yield significant results. The test reactions are evaluated visually and objectified by measuring the transepidermal water loss and possibly the degree of hydration and redness [60]. Details on the statistical analysis of the results of transepidermal water loss measurements can be found in the literature [67].

As the mentioned patch tests are usually performed in study participants with intact skin, the subjective tolerance should additionally be checked in participants with manifest or diagnosed rosacea. A suitable method, therefore, is the repeated open application test (ROAT) [68]. This test should preferably be performed on the face, as the rosacea-specific paresthesia occurs only there.

As for all other cosmetic products, the sensitizing potential of dermocosmetics for use in rosacea cannot be determined in human experiments for ethical reasons. To minimize the risk of contact sensitization, careful selection of the ingredients is recommended. Preservatives and other components with a high sensitizing potential should be abstained from, especially when alternatives with a lower sensitizing potential are available. 


\section{Product Documentation}

The information communicated on the packaging is usually not sufficient to estimate the quality of a product. Additional information should be documented by the manufacturer or the distributing company and made available to professionals.

This documentation should at least include the following:

1. A description of the galenic system, possibly specifying the $\mathrm{pH}$ value and the percentage of lipids and detergents.

2. The spectrum and amount of possible UV protection.

3. Proof of additional effects in the form of a synoptic statement describing the methods and testing institutions.
4. A summary of the results of the tolerance testing, describing the methods and testing institutions.

5. Specific application recommendations.

\section{Statement on the Procedure for Consensus Building}

The present guideline was written on behalf of the expert panel for dermocosmetics of the Society for Dermopharmacy (GD Gesellschaft für Dermopharmazie e.V.) as a consensus paper and its publication was authorized by the board of the GD.

\section{Disclosure Statement}

The authors of this guideline state no conflict of interests.

\section{References}

1 Reinholz M, Tietze JK, Kilian K, Schaller M, Schöfer H, Lehmann P, Zierhut M, Klövekorn W, Ruzicka T, Schauber J: Rosacea - S1 guideline. J Dtsch Dermatol Ges 2013;11:768-780.

2 Schauber J, Kresken J, Dürrfeld M, Hünerbein A, Kindl U, Klövekorn W, Merk HF, Reinholz M, Schaller M, Stelzner S: Interdisciplinary management of rosacea: statement of the Society for Dermopharmacy (in German). 2014. www.gd-online.de.

-3 Langenbruch AK, Beket E, Augustin M: Quality of health care of rosacea in Germany from the patient's perspective: results of the national health care study Rosareal 2009. Dermatology 2011;223:124-130.

-4 Augustin M, Herberger K, Hintzen S, Heigel H, Franzke N, Schäfer I: Prevalence of skin lesions and need for treatment in a cohort of 90,880 workers. Br J Dermatol 2011;165:865873.

5 Lehmann P: Rosacea: clinical features and classification (in German). Hautarzt 2013;64: 489-493.

6 Jansen T: Clinical presentations and classification of rosacea. Ann Dermatol Venereol 2011;138(suppl 3):192-200.

7 Akpek EK, Merchant A, Pinar V, Foster CS: Ocular rosacea: patient characteristics and follow-up. Ophthalmology 1997;104:18631867.

8 Awais M, Anwar MI, Iftikhar R, Iqbal Z, Shehzad N, Akbar B: Rosacea: the ophthalmic perspective. Cutan Ocul Toxicol 2015;34: 161-166.

-9 Steinhoff M, Schauber J, Leyden JJ: New insights into rosacea pathophysiology: a review of recent findings. J Am Acad Dermatol 2013; 69(suppl 1):15-26.
10 Cribier B: Pathophysiology of rosacea: redness, telangiectasia, and rosacea. Ann Dermatol Venereol 2011;138(suppl 3):184-191.

11 Yamasaki K, Gallo RL: The molecular pathology of rosacea. J Dermatol Sci 2009;55:77-81.

12 Schwab VD, Sulk M, Seeliger S, Nowak P, Aubert J, Mess C, Rivier M, Carlavan I, Rossio P, Metze D, Buddenkotte J, Cevikbas F, Voegel JJ, Steinhoff M: Neurovascular and neuroimmune aspects in the pathophysiology of rosacea. J Investig Dermatol Symp Proc 2011;15: 53-62.

13 Reinholz M, Ruzicka T, Schauber J: Cathelicidin LL-37: an antimicrobial peptide with a role in inflammatory skin disease. Ann Dermatol 2012;24:126-135.

14 Yamasaki K, Di Nardo A, Bardan A, Murakami M, Ohtake T, Coda A, Dorschner RA Bonnart C, Descargues P, Hovnanian A, Morhenn VB, Gallo RL: Increased serine protease activity and cathelicidin promotes skin inflammation in rosacea. Nat Med 2007;13: 975-980

15 Casas C, Paul C, Lahfa M, Livideanu B, Lejeune O, Alvarez-Georges S, Saint-Martory C, Degouy A, Mengeaud V, Ginisty H, Durbise E, Schmitt AM, Redoulès D: Quantification of Demodex folliculorum by PCR in rosacea and its relationship to skin innate immune activation. Exp Dermatol 2012;21:906-910.

16 Crawford GH, Pelle MT, James WD: Rosacea. 1. Etiology, pathogenesis, and subtype classification. J Am Acad Dermatol 2004;51:327341; quiz 342-344.

17 Cresce ND, Davis SA, Huang WW, Feldman SR: The quality of life impact of acne and rosacea compared to other major medical conditions. J Drugs Dermatol 2014;13:692-697.
18 van der Linden MM, van Rappard DC, Daams JG, Sprangers MA, Spuls PI, de Korte J: Health-related quality of life in patients with cutaneous rosacea: a systematic review. Acta Derm Venereol 2015;95:395-400.

19 Spoendlin J, Voegel JJ, Jick SS, Meier CR: A study on the epidemiology of rosacea in the UK. Br J Dermatol 2012;167:598-605

20 Kyriakis KP: Epidemiologic aspects of rosacea. J Am Acad Dermatol 2005;53:918-919.

21 Alexis AF: Rosacea in patients with skin of color: uncommon but not rare. Cutis 2010;86: 60-62.

22 van Zuuren EJ, Kramer SF, Carter BR, Graber MA, Fedorowicz Z: Effective and evidencebased management strategies for rosacea: summary of a Cochrane systematic review. $\mathrm{Br}$ J Dermatol 2011;165:760-781.

23 Belge K, Schaller M: Rosacea: current therapy options in focus (in German). Hautnah Derm 2013;1:27-33.

24 Schöfer H: Topical therapy of rosacea (in German). Hautarzt 2013;64:494-499.

25 Schaller M, Belge K: Systemic therapy of rosacea (in German). Hautarzt 2013;64:500-505.

26 Taieb A, Ortonne JP, Ruzicka T, Roszkiewicz J, Berth-Jones J, Peirone MH, Jacovella J: Superiority of ivermectin $1 \%$ cream over metronidazole $0.75 \%$ cream in treating inflammatory lesions of rosacea: a randomized, investigator-blinded trial. Br J Dermatol 2015;172: 1103-1110.

27 Fowler J Jr, Jackson M, Moore A, Jarratt M, Jones T, Meadows K, Steinhoff M, Rudisill D, Leoni M: Once-daily topical brimonidine tartrate gel $0.5 \%$ is a novel treatment for moderate to severe facial erythema of rosacea: results of two multicentre, randomized and vehiclecontrolled studies. Br J Dermatol 2013;166: 633-641. 
28 Del Rosso JQ, Preston NJ, Caveney SW, Gottschalk RW: Effectiveness and safety of modified release doxycycline capsules once daily for papulopustular rosacea monotherapy: results from a large community-based trial in subgroups based on gender. J Drugs Dermatol 2012;11:703-707.

29 Kerscher M, Reuther T: The curse of the Celts: cosmetic aspects in rosacea (in German); in Schöfer H (ed): Rosacea: Clinic and Current Therapy (in German). Stuttgart, Thieme, 2003, pp 67-72.

30 Borelli C, Korting HC: Non-pharmacologic management of rosacea (in German). Hautarzt 2011;62:828-833.

- 31 Draelos ZD, Colón LE, Preston N, Johnson LA, Gottschalk RW: The appearance of facial foundation cosmetics applied after metronidazole gel 1\%. Cutis 2011;87:251-259.

- 32 Levin J, Miller R: A guide to the ingredients and potential benefits of over-the-counter cleansers and moisturizers for rosacea patients. J Clin Aesthet Dermatol 2011;4:31-49.

33 Del Rosso JQ: Adjunctive skin care in the management of rosacea: cleansers, moisturizers, and photoprotectants. Cutis 2005;75: $17-21$.

34 Raab W, Kindl U: Care cosmetics (in German). Stuttgart, Wissenschaftliche Verlagsgesellschaft, 2012, p 347.

-35 Draelos ZD: The effect of Cetaphil gentle skin cleanser on the skin barrier of patients with rosacea. Cutis 2006;77(suppl 4):27-33.

- 36 Draelos Z, Hornby S, Walters RM, Appa Y: Hydrophobically modified polymers can minimize skin irritation potential caused by surfactant-based cleansers. J Cosmet Dermatol 2013;12:314-321.

37 Schmid-Wendtner MH, Korting HC: $\mathrm{pH}$ and Skin Care. Berlin, ABW, 2007.

38 Del Rosso JQ: The use of moisturizers as an integral component of topical therapy for rosacea: clinical results based on the assessment of skin characteristics study. Cutis 2009;84: $72-76$.

-39 Laquieze S, Czernielewski I, Baltas E: Beneficial use of Cetaphil moisturizing cream as part of a daily skin care regimen for individuals with rosacea. J Dermatol Treat 2007;18:158162.

-40 Seité S, Benech F, Berdah S, Bayer M, Veyrat S, Segot E, Sakalikova M, Gibejova L, Zelenkova $\mathrm{H}$ : Management of rosacea-prone skin: evaluation of a skin care product containing Ambophenol, Neurosensine, and La RochePosay thermal spring water as monotherapy or adjunctive therapy. J Drugs Dermatol 2013;8:920-924.

-41 Wu JJ, Weinstein GD, Kricorian GJ, Kormeili T, McCullough JL: Topical kinetin $0.1 \%$ lotion for improving the signs and symptoms of rosacea. Clin Exp Dermatol 2007;32:693-695.

-42 Vienne MP, Ochanda N, Borrel MT, Gall Y, Lauze C, Dupuy P: Retinaldehyde alleviates rosacea. Dermatology 1999;199(suppl 1):5356.
43 Weber TM, Ceilley RI, Buerger A, Kolbe L, Trookman NS, Rizer RL, Schoelermann A: Skin tolerance, efficacy, and quality of life of patients with red facial skin using a skin care regimen containing licochalcone A. J Cosmet Dermatol 2006;5:227-232.

44 Dupont E, Léveille C, Gomez J, Loigeret M, Loing E, Bilodeau D: Clinical efficacy of a serum integrating multiple cosmetic ingredients in the management of erythema of the face in aging skin. J Cosmet Dermatol 2012; 11:207-212.

45 Recommendation of the EU commission on 22 September 2006 about the efficacy of sun protection products and relating manufacturer information (2006/647/EG). Official J Eur Union 2006;265:39-43.

46 Seité S, Deshayes P, Dréno B, Misery L, Reygagne P, Saiag P, Stengel F, Roguedas-Contios A, Rougier A: Interest of corrective makeup in the management of patients in dermatology. Clin Cosmet Investig Dermatol 2012;5:123128.

47 Peuvrel L, Quéreux G, Brocard A, Saint-Jean M, Vallet C, Mère A, Labetoulle G, Le Fol C, Dréno B: Evaluation of quality of life after a medical corrective make-up lesson in patients with various dermatoses. Dermatology 2012; 224:374-380

48 Cosmetics Europe Recommendation: use of appropriate validated methods for evaluating sun product protection. 2013. www.cosmeticseurope.eu

49 Gehring W, Geier J, Gloor M: Studies on the dehydrating effect of various surfactants (in German). Dermatol Monatsschr 1991;177: 257-264.

50 Gloor M, Wasik B, Gehring W, Grieshaber R, Kleesz P, Fluhr JW: Cleansing, dehydrating, barrier-damaging and irritating hyperaemising effect of four detergent brands: comparative studies using standardized washing models. Skin Res Technol 2004;10:1-9.

51 Bornkessel A, Flach M, Arens-Corell M, Elsner P, Fluhr JW: Functional assessment of a washing emulsion for sensitive skin: mild impairment of stratum corneum hydration, $\mathrm{pH}$, barrier function, lipid content, integrity and cohesion in a controlled washing test. Skin Res Technol 2005;11:53-60.

52 Sonsmann FK, Strunk M, Gediga K, John C, Schliemann S, Seyfarth F, Elsner P, Diepgen TL, Kutz G, John SM: Standardization of skin cleansing in vivo. 1. Development of an Automated Cleansing Device (ACiD). Skin Res Technol 2014;20:228-238.

53 Jappe U, Schnuch A, Uter W: Rosacea and contact allergy to cosmetics and topical medicaments: retrospective analysis of multicentre surveillance data 1995-2002. Contact Dermatitis $2005 ; 52: 96-101$.

54 Jappe U, Schäfer T, Schnuch A, Uter W: Contact allergy in patients with rosacea: a clinicbased, prospective epidemiological study. J Eur Acad Dermatol Venereol 2008;22:12081214.
55 Scientific Committee on Consumer Safety: The SCCS's notes of guidance for testing of cosmetic ingredients and their safety evaluation. 2012. www.ec.europa.eu.

56 Cosmetics Europe: Cosmetic product test guidelines for the assessment of human skin compatibility. 1997. www.cosmeticseurope. eu

57 Walker AP, Basketter DA, Baverel M, Diembeck W, Matthies W, Mougin D, Röthlisberger R, Coroama M: Test guidelines for the assessment of skin tolerance of potentially irritant cosmetic ingredients in man. European Cosmetic, Toiletry and Perfumery Association. Food Chem Toxicol 1997;35:1099-1106; erratum in Food Chem Toxicol 1998;36:1.

58 Murahata RI, Nicoll GA: Mildness testing for personal washing products; in Aust LB (ed): Cosmetic Claims Substantiation. New York, Dekker, 1997, pp 153-169.

59 Tausch I, Bielfeldt S, Hildebrand A, Gassmüller J: Validation of a modified Duhring chamber test (DCT) as a repeated patch test. Parfümerie Kosmetik 1996;77:28-31.

60 Fischer T, Greif C, Wigger-Alberti W, Elsner $P$ : Instrumental methods to evaluate the safety and efficacy of cosmetics (in German). Aktuelle Derm 1998;24:243-250.

61 Frosch PJ, Kligman A: The Duhring chamber: an improved technique for epicutaneous testing of irritant and allergic reactions. Contact Dermatitis 1979;5:73-81.

62 Frosch PJ: Irritancy of soap and detergent bars; in Frosch PJ, Horwitz S (eds): Principles of Cosmetics for the Dermatologist. St Louis, Mosby, 1982, pp 5-12.

63 Nicoll GA, Murahata RI, Grove GL, Barrows J, Sharko PT: The relative sensitivity of two arm-wash test methods for evaluating the mildness of personal washing products. J Soc Cosmet Chem 1995;46:129-140.

64 Piérard GE, Goffin V, Piérard-Franchimont C: Corneosurfametry: a predictive assessment of the interaction of personal-care cleansing products with human stratum corneum. Dermatology 1994;189:152-156.

65 de Brugerolle de Fraissinette A, Picarles V, Chibout S, Kolopp M, Medina J, Burtin P, Ebelin ME, Osborne S, Mayer FK, Spake A, Rosdy M, De Wever B, Ettlin RA, Cordier A: Predictivity of an in vitro model for acute and chronic skin irritation (SkinEthic) applied to the testing of topical vehicles. Cell Biol Toxicol 1999;15:121-135.

66 Pittermann W, Kietzmann M: Bovine udder skin (BUS): testing of skin compatibility and skin protection (in German). ALTEX 2006; 23:65-71.

67 Kuss O, Diepgen TL: Proper statistical analysis of transepidermal water loss (TEWL) measurements in bioengineering studies. Contact Dermatitis 1998;39:64-67.

68 Hannuksela M, Salo H: The repeated open application test (ROAT). Contact Dermatitis 1986;14:221-227. 